\title{
Postoperative Management of Composite Tissue Graft Transplantation
}

\author{
Ayan Sen, Rula Al-Baghdadi, and Ali Al-Khafaji
}

\section{Introduction}

Composite Tissue Allotransplantation (CTA) involves transplantation of body structures such as the limbs, larynx, abdominal wall, tendons, and face. In this chapter, we will discuss general and specific postoperative management of patients following CTA.

\section{General Measures}

The following general preventative measures should be implemented:

\section{Elevation of the Head of the Bed}

A particular concern in the intensive care unit is ventilatorassociated pneumonia (VAP), defined as pneumonia that develops more than 48 after initiation of mechanical ventilation. Several preventative strategies have been examined to reduce the risk of VAP, including oropharyngeal decontamination, probiotics, silver coated endotracheal tubes, closed circuit suction tubes, and patient positioning. Elevation of the head of the bed is easy to implement without additional cost or adverse effects. The Center for Disease Control recommends that ventilated patients should ideally be placed in the semi-recumbent position with the head of the bed

A. Sen, M.B.B.S., M.Sc.

Mayo Clinic, Phoenix, AZ, USA

R. Al-Baghdadi, B.S. • A. Al-Khafaji, M.D., M.P.H. ( $\bowtie)$ Department of Critical Care Medicine, University of Pittsburgh School of Medicine, 3550 Terrace Street, 6th Floor, Scaife Hall, Pittsburgh, PA 15261, USA

e-mail: alkhafajia2@upmc.edu

(C) Springer Science+Business Media New York 2017

K. Subramaniam, T. Sakai (eds.), Anesthesia and Perioperative Care

for Organ Transplantation, DOI 10.1007/978-1-4939-6377-5_47 elevated at an inclination of $30-45^{\circ}$. Several randomized control trials have demonstrated that maintaining patients in the semi-recumbent position decreases the incidence of VAP.

\section{Venous Thromboembolism (VTE) Prophylaxis}

Development of venous thromboembolism is believed to evolve from abnormalities in endothelial injury, venous stasis, and hypercoaguability. Non-pharmacologic methods against VTE are available, though pharmacologic methods are preferred. Graduated compression stockings aim to prevent the pooling of blood in the lower extremities and prevent the development of venous clots. When appropriately used, compression stockings have been shown to decrease the rate of deep vein thrombosis. Pneumatic compression stockings applied to the lower extremities similarly operate by intermittently inflating and squeezing blood through the venous system to reduce the risk of venous stasis. Unfractionated heparin is most commonly used for prophylaxis against VTE. Both unfractionated heparin (UFH) and low molecular weight heparins (LMWH) have been shown to decrease the incidence of clinically detected VTE compared to placebo. Standard VTE prophylaxis, using compression stockings and pneumatic compression devices, should be used in all patients. Additionally, UFH can be used carefully in those without recent bleeding and without significant coagulopathy.

\section{Stress Ulcer Prophylaxis}

Stress related mucosal injury occurs in ICU patients within the first few days of ICU admission. The most important risk factors for the development of gastrointestinal bleeding are coagulopathy and need for mechanical ventilation. Other risk factors include history of gastrointestinal bleeding, hypotension, and multi-organ system dysfunction. Most of these risk factors are common in prior to and in the immediate 
postoperative period after transplant. Early trials of acid suppression used antacids, sucralfates, and $\mathrm{H} 2$ blockers and reported benefit in decreasing the incidence of both clinically significant and life-threatening gastrointestinal bleeding. Proton pump inhibitors (PPIs) are now commonly used as prophylactic measures against gastrointestinal bleeding. PPIs are more potent than $\mathrm{H} 2$ blockers in increasing gastric $\mathrm{pH}$ in critically ill patients; however, no study has shown improved efficacy with PPI to prevent gastrointestinal bleeding. Long-term PPI has been associated with increased risk of community acquired pneumonia, as well as healthcare associated pneumonia, and Clostridium Difficile infection.

\section{Specific Measures}

\section{Postoperative Intensive Care of the Face Transplant Patient}

Face transplantation is a complex and long surgery that is associated with significant perioperative challenges, and multiple postoperative complications. Face-grafting techniques require a well-defined organization of all the teams involved. Patients typically spend several days in the ICU.

\section{Airway Management}

Patients usually have a tracheostomy tube (size will be variable) in place. Many centers avoid the use of circumferential tracheostomy neck ties that normally fasten the tracheostomy tube. If these ties are used, tight application may lead to neck swelling and potentially venous outflow obstruction of the facial graft.

Postoperative severe graft edema is expected in most patients and head elevation at $30-45^{\circ}$ will promote venous drainage and prevent VAP [1]. Extra care must be taken to avoid airway loss because that complication has devastating consequences.

\section{Sedation}

The goal is to have all patients liberated from the mechanical ventilator within the first $24 \mathrm{~h}$ after surgery. Combinations of sedatives and narcotics are usually required. Great care should be taken to avoid over sedation. Sedation interruption should be implemented for patients who require ventilation longer than $24 \mathrm{~h}$.

\section{Ventilation}

Patients are ventilated with positive pressure volume controlled mode with $\mathrm{FiO} 2$ to keep paO2 levels between 100 and $150 \mathrm{mmHg}$ [1]. Pulse oxymetry is helpful to wean or titrate FiO2; however, arterial blood gases are generally required to guide manage acid base balance. The Risk of developing
Acute Lung Injury (ALI) and Acute Respiratory Distress Syndrome (ARDS) is high partly due to high operative transfusion requirement.

\section{Hemodynamics}

The goal is to have normal hemodynamics and avoid extreme swings (high or low) in blood pressure and heart rate as much as possible.

Hypothermia, hypovolemia, and pain can cause vasoconstriction, which in turn may compromise the free flaps. Normothermia, mean arterial pressure (MAP) of $65 \mathrm{mmHg}$, urine output up to $0.5-1 \mathrm{ml} / \mathrm{kg} / \mathrm{h}$ should be the goal. Graft perfusion monitoring is performed by Doppler velocities of the external carotid artery but continuous Doppler probes may be used.

Hypotension and shock can be hypovolemic, hemorrhagic, or vasodilatory and the management should be implemented accordingly. There is no evidence to suggest using one vasopressor or inotrope is better than another so local protocols in managing shock should be utilized.

\section{Volume Status}

Graft edema is expected because of the long operation, long ischemia time, and disrupted lymphatic drainage. Elevation of the head $30-45^{\circ}$ and relatively negative fluid balance can used to reduce face edema. Use of small does of diuretics may be used if deemed necessary by the team.

\section{Massive Transfusion}

Large blood losses are expected intraoperatively due to high vascularity of the face and prolonged procedure time as a result of complex anastomoses during the preparation and grafting phase. Maintaining Hct $>27$, Plt $>50 \mathrm{~K}$, INR $<1.5$ and Fibrinogen $>100$ are common clinical practice targets [2].

\section{Prophylaxis}

Venous thromboembolism (VTE) prophylaxis with lowmolecular-weight or unfractionated heparin are implemented as long as there is no evidence of bleeding. $\mathrm{H} 2$ blockers or proton pump inhibitors are frequently used for stress ulcers prophylaxis.

\section{Enteral Feeding}

Most patients have a gastrostomy or jejunostomy tube and Enteral feeding should be started in all patients usually on the first postoperative day. High protein formulas are used to help ensure adequate healing $(25-30 \mathrm{kcal} / \mathrm{kg} / \mathrm{day}$ and $1.5-$ $2.0 \mathrm{~g}$ of protein $/ \mathrm{kg} /$ day), and water needs at $25-30 \mathrm{ml} / \mathrm{kg} /$ day. In our institution, IMPACT 1.5 has been the formula of choice in free flap patients. Periodic speech-and-swallow evaluations are performed after the first $24 \mathrm{~h}$ to assess safe oral feeding. 


\section{Vascular Access}

Femoral venous lines are placed for the perioperative use but should be discontinued as soon as possible. If indwelling catheters are needed for longer duration, peripherally inserted central catheters (PICC) may be considered.

\section{Infections and Antibiotics}

Patients generally receive perioperative broad spectrum antibiotic prophylaxis and the choice depends on the local institution microbiological resistance pattern. Early infections if it occurs are mostly bacterial. The risk of infection mandates early removal of all invasive lines and subsequent frequent screening. The most severe infections occur during times of over-immunosuppression, such as the induction phase or management of acute rejection. Cytomegalovirus (CMV), Epstein Barr virus (EBV), and cutaneous herpes simplex infections have been reported [1]. CMV can trigger rejection that can be challenging to control; therefore, ganciclovir must be administered based on donor and recipient data. Candida albicans infection has also been reported. Prophylaxis with Ganciclovir as well as antifungals like fluconazole and Bactrim for pneumocystis should be considered. Infectious disease specialists should be involved in tailoring antibiotics to the results of surveillance cultures from both the donor and the recipient [3].

\section{Immunosuppression}

Proper levels of immunosuppression should minimize the risks of opportunistic infectious complications. A sentinel skin graft is placed sometimes in the left infra-mammary area to monitor rejection episodes [1]. Acute rejection has been reported as early as 4 days postgrafting. Induction immunosuppression is achieved with anti-thymocyte globulin as well as steroid taper. Maintenance immunosuppression involves a regime of tacrolimus, mycophenolate mofetil, and prednisone, and must be closely followed up for the duration of the patient's or allograft's life. Over-immunosuppression leads to undesirable side effects, such as infections, while under-immunosuppression leads to rejection of the allograft. Blood levels of immunosuppression medications need to be monitored, especially calcineurin inhibitors which are associated with significant nephrotoxicity, and dosages must be adjusted constantly to prevent deleterious side effects, while maintaining drug efficacy.

\section{Rehabilitation}

Social workers provide postoperative support on an as needed basis. They typically meet with the patient every 1-2 days during the acute postoperative period. The team's psychiatrist meets daily with the FT recipient during his/her perioperative hospitalization. After discharge, the psychiatrist meets weekly with the recipient, or on an as needed basis. Rehabilitation is crucial to the attainment of maximum motor recovery during the postoperative period. The rehabilitation protocol is created and adjusted for each patient and must be started as early as possible. The initial focus is on mobility and airway clearance, and soon after, therapeutic exercise.

\section{Upper Limb Transplant}

\section{Airway Management}

There is no special airway management described for patients with upper limb transplant. Risk of ARDS from massive transfusion and postoperative infections are possible. Patients are sedated and ventilated until they are hemodynamically stable and there is no acute concern about the graft function. Usually they are extubated in the first $24 \mathrm{~h}$ after surgery.

\section{Hemodynamics}

Central venous catheter is usually placed in the internal jugular vein to be used not only for access but also to obtain central venous pressure (CVP) and mixed oxygen saturation (SVO2). An additional 14-gauge IV catheter is recommended in the nonoperative arm for a unilateral hand transplant. Hemodynamic monitoring by a 20 -gauge radial artery cannula in the nonoperative arm or an 18-gauge femoral artery cannula for bilateral hand transplant recipients is undertaken.

Similar to face transplant, vasoconstriction caused by hypothermia, hypovolemia, and pain could have a negative impact on the free flap [4]. Circulatory monitoring should be continued in the ICU to maintain mean Arterial pressure between 60 and $65 \mathrm{mmHg}$. Alpha- agonists should be avoided as they may affect graft perfusion. Dopamine is instituted when hypotension is not adequately corrected by infusion of IV fluids or blood products, such as vasodilation in response to donor extremity reperfusion. Flap/replantation assessment includes capillary refill and pulse check every hour as well as hourly monitoring via the implanted Doppler and two pulse oximeters. In unilateral transplants, it is crucial to compare the waveforms and oxygen saturations between the new graft and the non-transplant contralateral control limb [4, 5]. In bilateral transplants, this becomes a more challenging exercise and even subtle changes should be documented and reported. The transplanted limb is elevated, warm air blankets cover the limb, and the room is kept warm at $75{ }^{\circ} \mathrm{F}$ to prevent vasoconstriction.. Pain management includes continuous nerve block(s), acetominophine, and intravenous narcotics as needed. 


\section{Transfusion Requirements}

Similar to face transplant, large volume of blood product transfusion may be necessary. The Pittsburgh protocol follows the trauma resuscitation protocol of 1 unit packed red blood cells (PRBC): 1 unit fresh frozen plasma (FFP): $250 \mathrm{cc}$ normal saline [6]. This ratio achieves a hematocrit of 26-28\% [7]. Other parameters are similar to the face transplant management.

\section{Volume Status}

Close monitoring of volume status by trends in central venous pressure and hourly urine output is essential. Graft edema in the postoperative phase is managed conservatively by elevation and compressive bandaging. If venous stasis or lymphedema is suspected clinically, appropriate investigations (venograms or lymphangiograms) are performed to exclude these conditions. Limb volumetry is performed during the early therapy phase to identify and monitor subtle volume change [4]. Risk of acute kidney injury is high due to a combination of events including the lengthy surgery, the release of endogenous nephrotoxins from muscles and tissues, and the initiation of immunosuppressive drugs.

\section{Prophylaxis}

Deep vein thrombosis prophylaxis with low molecular weight heparin and stress ulcer with proton pump inhibitors is continued.

\section{Immunosuppression}

Immunosuppression is induced by a specific medication regimen. Postoperative immunosuppressions have included induction with antithymocyte globulin in some patients and basiliximab in others. Maintenance immunosuppression can be achieved by any of the following:

- Tacrolimus, mycophenolate, and corticosteroids.

- Tacrolimus and steroids.

- Rapamycin and mycophenolate.

- Rapamycin with topical steroid and tacrolimus.

Patient survival is $100 \%$. The first hand transplant recipient lost his graft due to rejection from noncompliance. Five other patients lost their grafts due to inability to continue immunosuppression $[8,9]$.

Skin and muscle biopsies are performed per institutional protocol to assess for evidence of acute rejection such as endpoint edema, erythema, and necrosis. Acute rejection was seen in some patients, most of them between 7 and 14 weeks posttransplantation. All the episodes were reversed with the use of intravenous steroids/lymphocyte depleting agents, alemtuzumab [10] or ATG/basiliximab and/or topical tacrolimus/corticosteroid.

\section{Immunosuppression}

The University of Pittsburgh Medical Center (UPMC) developed and implemented a new protocol based on the hypothesis that immunomodulation with donor bone marrow cell-based therapies would reduce long-term immunosuppression requirements $[4,6,8]$. There are three components to the "Pittsburgh Protocol": induction therapy with Alemtuzumab (30 mg), tacrolimus monotherapy $(0.2 \mathrm{mg} / \mathrm{kg} /$ day) at $12 \mathrm{~h}$ with an initial target trough level of $10-12 \mathrm{ng} /$ $\mathrm{ml}$, and whole bone marrow cells infused on day 14 .

The protocol is efficacious and well tolerated, and episodes of acute rejection are low-grade and infrequent. Functional, immunologic, and graft survival outcomes continue to be assessed during long-term follow-up.

\section{Infection}

All patients receive prophylactic antibiotics, antifungals, and antivirals similar to patients with face transplant. Risk of postoperative pneumonia, CMV, and other infections like cutaneous mycosis and ulnar osteitis due to Staphylococcus aureus have been reported and need to be managed aggressively. Graft failure can occur due to postoperative infections.

\section{Other Complications}

Early postoperative complications include vascular thrombosis, limb loss, bleeding, and deep venous thrombosis (DVT). Re-exploration of the anastomosis may be necessary. Hip osteonecrosis has also been reported in long-term follow-up due to steroid use. Potential complications of prolonged immunosuppression as well as increased risk of malignancy, nephrotoxicity, neurotoxicity, gastrointestinal toxicity, hypertension, and diabetes, which are all related to the immunosuppressive medication. No graft versus host disease, no chronic rejection, and no malignancies or life-threatening conditions due to hand transplantation were reported. Hand graft survival rates have been higher than other forms of solid organ transplants [11]. Management of graft failure involves amputation followed by use of prosthesis or another transplant.

\section{Rehabilitation}

Postoperatively, electrical muscle stimulation may improve tendon gliding; therefore, encore electrodes should be placed along the median and ulnar nerves (above the transplant), along with a transcutaneous electrical nerve stimulator [8]. The patient should receive physical and occupational therapy daily for at least the first postoperative week.

Involvement of physical therapists and rehabilitation consultants is aimed to achieve optimal outcomes. The postoperative psychotherapy should be enhanced or reduced in accordance with the patient's history of compliance with health care needs, as determined by the social worker and psychiatrist preoperatively. 


\section{References}

1. Sedaghati-Nia A. Top of form anaesthesia and intensive care management of face transplantation. Br J Anaesth. 2013;111(4):600-6.

2. Cabrales P, Intaglietta M, Tsai AG. Transfusion restores blood viscosity and reinstates microvascular conditions from hemorrhagic shock independent of oxygen carrying capacity. Resuscitation. 2007;75:124-34.

3. Bonatti H, Brandacher G, Margreiter R, Schneeberger S. Infectious complications in three double hand recipients: experience from a single center. Transplant Proc. 2009;41:517-20.

4. Lovasik D, Foust DE, Losee JE, Lee WP, Brandacher G, Gorantla VS. Helping hands: caring for the upper extremity transplant patient. Crit Care Nurs Clin North Am. 2011;23(3):505-17.

5. Azari KK, Imbriglia JE, Goitz RJ, et al. Technical aspects of the recipient operation in hand transplantation. Transplant Proc 2009;41(2):472-5.
6. Lang RS, Gorantla VS, Esper S, Montoya M, Losee JE, Hilmi IA, Sakai T, Lee WP, Raval JS, Kiss JE, Shores JT, Brandacher G, Planinsic RM. Anesthetic management in upper extremity transplantation: the Pittsburgh experience. Anesth Analg. 2012;115(3):678-88.

7. Sperry JL, Ochoa JB, Gunn SR, et al. A FFP:PRBC transfusion ratio $1: 1.5$ is associated with a lower risk of mortality following massive transfusion. J Trauma. 2008;65:986-9.

8. Amirlak B, Gorantla VS, Gonzalez NR, et al. Hand transplantation. http://www.emedicine.medscape.com/article/1370502-overview.

9. Schneeberger S, Gorantla VS, Hautz T, et al. Immunosuppression and rejection in human hand transplantation. Transplant Proc. 2009;41(2):472-5.

10. Schneeberger S, Landin L, Kaufmann C, et al. Alemtuzumab: key for minimization of maintenance immunosuppression in reconstructive transplantation? Transplant Proc. 2009;41(2): 499-502.

11. Petruzzo P, Lanzetta M, Dubernard JM, et al. The international registry on hand and composite tissue transplantation. Transplantation. 2010;90(12):1590-4. 